03

\title{
Формирование трехмерных униполярных импульсов при движении зарядов в вакууме
}

\author{
(C) Н.Н. Розанов ${ }^{1,2,3}$ \\ ${ }^{1}$ Государственный оптический институт им. С.И. Вавилова, \\ 199053 Санкт-Петербург, Россия \\ ${ }^{2}$ ФТИ им. А.Ф. Иофрфе, \\ 194021 Санкт-Петербург, Россия \\ ${ }^{3}$ Университет ИТМО, \\ 197101 Санкт-Петербург, Россия \\ e-mail: nnrosanov@mail.ru
}

Поступила в редакцию 11.09.2019 г.

В окончательной редакции 11.09.2019 г.

Принята к публикации 20.09.2019 г.

\begin{abstract}
При использовании аналогии с задачами электростатики в общем виде решена задача о пространственном (трехмерном) распределении электрической площади импульсов излучения, определяемой как интеграл по времени от напряженности электрического поля, при генерации излучения движением электрических зарядов в вакууме. Продемонстрирована возможность формирования униполярных импульсов излучения, у которых основная поляризационная составляющая не меняет знак в течение импульса.
\end{abstract}

Ключевые слова: уиполярные импульсы излучения, электрическая площадь импульсов.

DOI: $10.21883 /$ OS.2020.01.48843.258-19

Прогресс лазерной физики и техники делает актуальной задачу получения все более коротких импульсов излучения, вплоть до субцикловых и квазиуниполярных, у которых основная поляризационная компонента напряженности электрического поля содержит существенную постоянную составляющую. Такие импульсы без осцилляций напряженности электрического поля оказывают более сильное воздействие на классические и квантовые микрообъекты, чем стандартные биполярные, напряженность электрического поля у которых осциллирует [1-9]. Имеется ряд методов генерации квазиуниполярных импульсов излучения [10-12] и экспериментальные подтверждения существования таких импульсов [13,14]. Однако предложенные схемы преимущественно используют одномерное приближение и потому актуальна задача формирования трехмерных пакетов униполярного излучения. Задачей данной работы служит анализ возможности формирования подобных пакетов за счет движения заряженных частиц в вакууме.

Исходными здесь служат два уравнения Максвелла для электромагнитного поля в вакууме с зарядами $[15,16]$ :

$$
\operatorname{rot} \mathbf{E}=-\frac{1}{c} \frac{\partial \mathbf{H}}{\partial t}
$$

и

$$
\operatorname{div} \mathbf{E}=4 \pi \rho .
$$

В (1) и (2) $\mathbf{E}$ и $\mathbf{H}-$ напряженности электрического и магнитного поля соответственно, $c$ - скорость света в вакууме, $t-$ время и $\rho-$ плотность электрических зарядов. Для пакетов излучения с конечной энергией интегрирование (1) по времени в бесконечных пределах приводит к соотношению (см. [17-20] и приведенные там ссылки):

$$
\operatorname{rot} \mathbf{S}_{E}=0,
$$

где введена электрическая площадь импульса излучения $\mathbf{S}_{E}=\int_{-\infty}^{\infty} \mathbf{E} d t$. Аналогично, проинтегрировав по времени (2), получим

$$
\operatorname{div} \mathbf{S}_{E}=4 \pi Q
$$

В (5) $Q(\mathbf{r})=\int_{-\infty}^{+\infty} \rho(\mathbf{r}, t) d t-$ плотность заряда, протекшего через окрестность точки $\mathbf{r}=(x, y, z)$ за весь бесконечный промежуток времени (предполагается конечность этой величины).

Уравнения (3) и (4) формально совпадают с основными уравнениями электростатики $[15,16]$ при замене $\mathbf{E} \rightarrow \mathbf{S}_{E}$ и $\rho \rightarrow Q$. Это позволяет заимствовать из электростатики решение этих уравнений, т.е. определение площади электрического поля $\mathbf{S}_{E}$ по заданному распределению плотности заряда $Q$. А именно ввиду (3)

$$
\mathbf{S}_{E}=-\operatorname{grad} \Phi_{S},
$$

где „потенциал“ $\Phi_{S}$ с учетом (4) подчиняется уравнению Пуассона

$$
\Delta \Phi_{S}=-4 \pi Q .
$$

Решением (6) служит ньютонов потенциал

$$
\Phi_{S}(\mathbf{r})=\int \frac{Q\left(\mathbf{r}^{\prime}\right)}{\left|\mathbf{r}-\mathbf{r}^{\prime}\right|} d \mathbf{r}^{\prime} .
$$

Уравнения (5) и (7) решают в общем виде поставленную задачу. 
Представим, что распределение поля создается пучком заряженных частиц (электронов), влетающих в полупространство $z>0$ через малое отверстие в металлическом экране, расположенном при $z=0$, и через некоторое время покидающих это полупространство. Другим вариантом служит отрыв электронов из металла импульсом ионизирующего излучения с последующей рекомбинацией зарядов на том же или другом экране. Для идеального металла граничное условие при $z=0$, по аналогии с электростатикой, заключается в требовании, чтобы плоскость $z=0$ была эквипотенциальной поверхностью для „потенциала“ $\Phi_{S}$; без ограничения общности можно положить $\Phi_{S}(z=0)=0$. Тогда задача может быть решена методом изображений [16]. Введем плотность дополнительных фиктивных зарядов $\bar{Q}$, расположенных симметрично относительно плоскости $z=0$ по отношению к реальным и обладающих противоположным знаком заряда: $\bar{Q}(\overline{\mathbf{r}})=-Q(\mathbf{r})$. При этом, если $\mathbf{r}=(x, y, z)$, то $\overline{\mathbf{r}}=(x, y,-z)$. Тогда

$$
\begin{aligned}
\Phi_{S}(\mathbf{r})= & \int \frac{Q\left(\mathbf{r}^{\prime}\right)}{\left|\mathbf{r}-\mathbf{r}^{\prime}\right|} d \mathbf{r}^{\prime}+\int \frac{\bar{Q}\left(\overline{\mathbf{r}}^{\prime}\right)}{\left|\mathbf{r}-\overline{\mathbf{r}}^{\prime}\right|} d \overline{\mathbf{r}}^{\prime} \\
& =\int_{z^{\prime}>0} Q\left(\mathbf{r}^{\prime}\right)\left(\frac{1}{\left|\mathbf{r}-\mathbf{r}^{\prime}\right|}-\frac{1}{\left|\mathbf{r}-\overline{\mathbf{r}}^{\prime}\right|}\right) d \mathbf{r}^{\prime} .
\end{aligned}
$$

Поскольку $z>0, z^{\prime}>0$ и

$$
\begin{aligned}
& \left|\mathbf{r}-\mathbf{r}^{\prime}\right|=\left[\left(x-x^{\prime}\right)^{2}+\left(y-y^{\prime}\right)^{2}+\left(z^{2}-2 z z^{\prime}+z^{\prime 2}\right)\right]^{1 / 2}< \\
& \quad<\left[\left(x-x^{\prime}\right)^{2}+\left(y-y^{\prime}\right)^{2}+\left(z^{2}+2 z z^{\prime}+z^{\prime 2}\right)\right]^{1 / 2}=\left|\mathbf{r}-\overline{\mathbf{r}}^{\prime}\right|,
\end{aligned}
$$

подынтегральная функция в последнем выражении в (8) имеет тот же знак, что и $Q$. Поэтому при инжектировании зарядов одного знака (электронов) подынтегральная функция знакопостоянна (знак тот же, что у зарядов) и „потенциал“ $\Phi_{S}$ отличен от нуля. Тем самым при расположении микрообъекта в области максимального по модулю градиента „потенциала“ микрообъект будет испытывать максимальное воздействие униполярного импульса излучения, генерируемого движением зарядов. Естественно, что время воздействия импульса излучения на объект, предварительно расположенный в области значительного градиента „потенциала“, не может превышать время пребывания зарядов в полупространстве $z>0$.

Таким образом, аналогия с электростатикой позволяет в общем виде решить задачу о распределении электрической площади импульсов излучения, генерируемого в вакууме за счет движения электрических зарядов. К классическим (некорректным) обратным задачам теории потенциала [20,21] принадлежит также задача об определении плотности заряда $Q$ по желательному распределению „потенциала“ $\Phi_{S}$, которая сводится к решению интегрального уравнения. Результаты такого анализа имеют существенное значение для разработки схем с максимальной по абсолютной величине электрической площадью.

\section{Финансирование работы}

Исследование поддержано Программой президиума РАН „Математика и нелинейная динамика“ и грантом РФФИ № 19-02-00312.

\section{Конфликт интересов}

Автор заявляет, что у него нет конфликта интересов.

\section{Список литературы}

[1] Dimitrovski D., Solov'ev E.A., Briggs J.S. // Phys. Rev. Lett. 2004. V. 93. P. 083003.

[2] Dimitrovski D., Solov'ev E.A., Briggs J.S. // Phys. Rev. A. 2005. V. 72. P. 043411.

[3] Song X., Yang $W$., Zeng Z., Li R., Xu Z. // Phys. Rev. A. 2010. V. 82. P. 053821.

[4] Розанов Н.Н. // Опт. и спектр. 2018. Т. 124. № 1. С. 75; Rosanov N.N. // Opt. Spectrosc. 2018. V. 124. N 1. P. 72.

[5] Hassan M.T., Luu T.T., Moulet A., Raskazovskaya O. et al. // Nature. 2016. V. 530. P. 66.

[6] Chai X., Ropagnol X., Mohsen Raeis-Zadeh S., Reid M., Safavi-Naeini S., Ozaki T. // Phys. Rev. Lett. 2018. V. 121. P. 143901.

[7] Розанов Н.Н., Архипов Р.М., Архипов М.В. // УФН. 2018. T. 188. № 12. C. 1347-1353; Rosanov N.N. Arkhipov R.M., Arkhipov M.V. // Phys. Usp. 2018. V. 61. N 12. P. 1227-1233.

[8] Розанов Н.Н. // Опт. и спектр. 2019. Т. 126. № 2. C. 211-213; Rosanov N.N. // Opt. Spectrosc. V. 126. N 2. P. $140-143$.

[9] Розанов Н.Н., Архипов М.В., Архипов Р.М., Веретенов Н.А., Пахомов А.В., Федоров С.В. // Опт. и спектр. 2019. T. 127. № 1. C. 82-93; Rosanov N.N., Arkhipov M.V., Arkhipov R.M., Veretenov N.A., Fedorov S.V. // Opt. Spectrosc. 2019. V. 127. N 1. P. 77.

[10] Wu H.-C., Meyer-ter-Vehn J. // Nature Photon. 2012. V. 6. P. 304.

[11] Xu J., Shen B., Zhang X., Shi Y., Ji L., Zhang L., Xu T., Wang W., Zhao X., Xu Z. // Sci. Rep. 2018. V. 8. P. 2669 (2018).

[12] Архипов Р.М., Пахомов А.В., Архипов М.В., Бабушкин И., Толмачев Ю.А., Розанов Н.Н. // Письма в ЖЭТФ. 2017. T. 105. № 6. C. 388; Arkhipov R.M., Pakhomov A.V., Babushkin I., Tolmachev Yu.A., Rosanov N.N. // JETP Letters. 2017. V. 105. N 6. P. 408.

[13] Naumenko G., Shevelev M. // J. Instrumentation. 2018. V. 13. № 5. P. C05001.

[14] Hassan M.T., Luu T.T., Moulet A., Raskazovskaya O., Zhokhov P., Garg M., Karpowicz N., Zheltikov A.M., Pervak V., Krausz F., Goulielmakis E. // Nature. 2016. V. 530. P. $66-70$.

[15] Ландау Л.Д., Лифиии, Е.М. Теория поля. М.: Наука, 1988; Landau L.D., Lifshitz E.M. The Classical Theory of Fields. Oxford: Pergamon Press Ltd. 1975.

[16] Ландау Л.Д., Лифиии Е.М. Электродинамика сплошных сред. М.: Наука, 1982; Landau L.D., Lifshitz E.M., Pitaevskii L.P. Electrodynamics of Continuous Media. Oxford: Butterworth-Heinemann, 1984. 
[17] Розанов Н.Н. // Опт. и спектр. 2009. Т. 107. № 5. C. 761-765; Rosanov N.N. // Opt. Spectrosc. 2009. V. 107. N 5. P. $721-725$.

[18] Rosanov N.N., Kozlov V.V., Wabnitz S. // Phys. Rev. A. 2010. V. 81. N 4. P. 043815.

[19] Розанов Н.Н. Диссипативные оптические солитоны. М.: Физматлит, 2011.

[20] Тихонов А.Н. // Докл. АН СССР. 1943. Т. 39. № 5. C. $195-198$.

[21] Лаврентьев М.М., Романов В.Г., Шишатский С.П. Некорректные задачи математической физики и анализа. М.: Наука, 1980. 